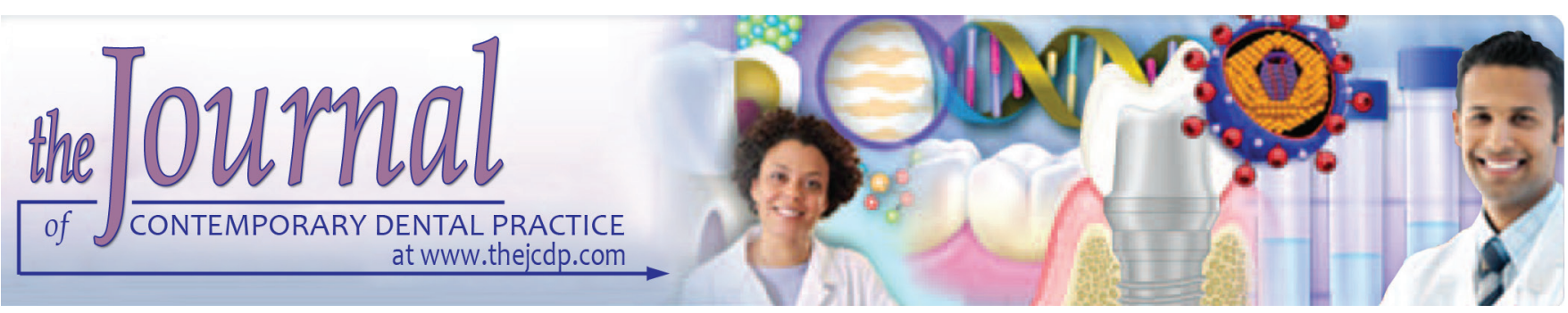

\title{
Effect of the Curing Temperature of Dental Composites evaluated with a Fluorescent Dye
}

\author{
${ }^{1}$ Maciel E da Silva-Júnior, ${ }^{2}$ Rosane de FZ Lizarelli, ${ }^{3}$ Vanderlei S Bagnato, ${ }^{4}$ Mateus R Tonetto, ${ }^{5}$ Flávio Simões \\ ${ }^{6}$ Álvaro H Borges, ${ }^{7}$ Matheus C Bandéca, ${ }^{8}$ Marcelo F de Andrade
}

\begin{abstract}
Aim: With the development of the light-emitting diode (LED) to photo-activate composite resin, greater intensities could be reached without greater elevation of temperature in the mass of the composite resin and in the dental structure arisen from the irradiance in comparison to halogen equipments. This new scenario created a necessity to investigate the influence of temperature over the composite polymerization.
\end{abstract}

Materials and methods: Several curing temperatures (Tcure$0,25,50,75$, and $100^{\circ} \mathrm{C}$ ) were used to polymerize a composite resin (Filtek Z250, 3M ESPE) for 40 and $60 \mathrm{~s}$, using the halogen equipment Gnatus Optilight Digital (halogen) and two LEDs that use a new technology to assembly the diodes: LEC 1000 and bright LEC (MM Optics) (LED 1 and LED 2 respectively). The influence of curing temperature, added by the other variables studied, was evaluated using a methodology developed and improved at IFSC/USP, in which the penetration of a fluorescent dye in the body of the photopolymerized composite resin was quantified using fluorescence spectroscopy.

Results: According to the final data submitted to an analysis of variance, the presence of two groups of results could be verified: Between 0 and $25^{\circ} \mathrm{C}$, both had a great percentage of the dye penetration compared with other Tcure with a variation in penetration from $69.26 \pm 8.19 \%$ to $90.99 \pm 3.38 \%$. In this analysis, the effects of time and temperature were highly notable $(p<0.05)$ and the lesser value of dye penetration took place at 60 s of photoactivation This penetration was, in average, smaller with the Tcure of $25^{\circ} \mathrm{C}$. The results showed that there was an

\footnotetext{
${ }^{1,8}$ Department of Restorative Dentistry, Araraquara School of Dentistry, UNESP_Universidade Estadual Paulista, São Paulo, Brazil

2,3 Department of Physics, USP_Universidade de São Paulo São Carlos, Brazil

${ }^{4-7}$ Department of Postgraduate Program in Dentistry, University of Cuiabá, Cuiabá, Mato Grosso, Brazil
}

Corresponding Author: Maciel E da Silva-Júnior, St. Humaitá 1680 Araraquara, 14801903, São Paulo, Brazil, e-mail: macieljr@hotmail.com interaction between the equipment and time and between time and temperature; the other group is regarding the Tcure was from 50,75 , and $100^{\circ} \mathrm{C}$, despite the $p=0.05$, the effect of temperature was notable. The penetration of the dye ranged from $8.87 \pm 3.55$ to $39.47 \pm 8.9 \%$. The effects of equipment and time were highly notable. The penetration with the time of $60 \mathrm{~s}$ was in average smaller. Except with the equipment LED 1, the percentages of the dye penetration were greater with the Tcure of $100^{\circ} \mathrm{C}$. The smallest average was the Tcure of $50^{\circ} \mathrm{C}$ and $60 \mathrm{~s}$ of photoactivation.

Conclusion: Based on the available data regarding the influence of curing temperature on the polymerization process of composite resins, was possible to concluded that small increments of heat increased the degree of conversion. We can assume that the energy supply through the generation of heat by the photopolymerizing devices can function as a heating medium for the reagent system by reducing its viscosity and increasing the mobility and agitation of its components.

Clinical significance: The dentist must be aware of the effects that exist between the activation devices on the light output and their heat transmission to the composite and the tooth itself. This heat transmission might create a polymer with better characteristics.

Keywords: Diffusion, Fluorescence, Heat transference, Polymers, Visible light.

How to cite this article: da Silva-Júnior ME, de FZ Lizarelli R, Bagnato VS, Tonetto MR, Simões F, Borges ÁH, Bandéca MC, de Andrade MF. Effect of the Curing Temperature of Dental Composites evaluated with a Fluorescent Dye. J Contemp Dent Pract 2018;19(1):3-12.

Source of support: Nil

Conflict of interest: None

\section{INTRODUCTION}

Certain characteristics, especially those of halogen lamps, such as broader emission spectrum, low durability, and increased heat generation, have made light sources based on LEDs a good option for photopolymerization of composite resins. The LED photopolymerizers have several characteristics that make them extremely viable for the curing of composite resins, such as: Longer life with about $100,000 \mathrm{~h}$ of durability, light emission only in the optimum 
absorption region of the initiator most commonly used in composite resins, and absence of heat irradiance. ${ }^{1-5}$ This latter characteristic has been posed as one of the greatest advantages for the clinician since the absence of wavelength emission in the infrared range prevents excessive heating in the long-term procedures, which could result in damages to the biological structures. ${ }^{3}$

There is, however, an important point that has not been considered in this technological transformation of photoactivation: The influence of temperature on the final properties of the photopolymerized composite resin.

The temperature factor may affect polymerization kinetics in two different situations. One would be the curing temperature, which is the temperature at which the monomers are at the time of polymerization. Moreover, another situation would be the heat of polymerization, i.e., the generation of heat due to the process of photoactivation of the composite resin..$^{6,7}$

Even though the influence of heat generation by the photo activation light source was the main concern of this research, the whole experiment was carried out using the curing temperature variation, which depends on the external environment and not on the irradiance of the apparatus or the conversion of the composite resin. At the outset, in our view, understanding how the curing temperature influences the polymerization process would allow a better understanding of this modification imposed by the use of a photopolymerization source that raises the temperature less during its operation. ${ }^{8}$

Like any chemical reaction, the polymerization is also affected depending on the thermal agitation present in the molecules that constitute them. Therefore, temperature variations during the curing process may modify the properties of the final cured resin and its molecular arrangement. This new molecular organization, due to the alteration of the curing temperature, can generate a polymer with different incorporation characteristics of pigments on its surface. This last aspect is of special relevance in view of the role of photopolymerizable composite resins in esthetic restorative dentistry. However, as in research conducted by Wu, ${ }^{5}$ De Gee et al, ${ }^{6}$ and Andrade, ${ }^{7}$ this penetration of molecules can be used as a method to evaluate the polymerization of these composite resins, because the diffusion rate of penetrants depends on the free volume of the polymer formed, and this free volume depends on the form of polymerization and degree of conversion. ${ }^{6-9}$

Considering the probable influence of the thermal energy supply on the photopolymerization process, we will investigate, through a different methodology from the traditional evaluation methods, such as degree of conversion [Fourier transform infrared (FTIR)] and microhardness, the consequences of the temperature variation during curing through the quantification of the penetration of an organic and fluorescent dye into the mass of the composite resin photopolymerized by two LEDs and a halogen lamp.

We can consider that the cure temperature (Tcure) of a polymer is the temperature at which the monomer mass is just at before the start of the polymerization, regardless of whether the process is exclusively chemical or photoactivated. As the reaction of monomers into polymers is an exothermic process, a further increase of heat will occur proportional to the rate of reaction and the degree of polymerization. This increase will also be elevated even more due to the irradiance of the light used, ${ }^{8}$ which becomes another modifying factor of the kinetics of cure. We can then verify the occurrence of two distinct situations in which the temperature change can influence the photopolymerization process. Both have distinct, but nonindependent consequences.

According to Watts, ${ }^{9}$ there is always an increase in the propagation rate of the reaction as the monomeric conversion increases (self-acceleration). This is related with an elevation in temperature of the reagent medium and a higher viscosity of the forming molecules.

The heat generation, its magnitude, and its duration will probably influence the curing kinetics, the mechanical properties, and the morphology of the polymers.

One phenomenon that must be considered is the thermal diffusivity (a rate at which a thermal equilibrium is reached by a body) because it allow us to characterize the transient thermal response of a restoration undergoing a temporary thermal stimulus. ${ }^{10}$

For the enamel, the diffusivity is $0.0042 \mathrm{~cm}^{2} / \mathrm{s}$ and is much larger than that of the dentin, which is 0.0026 $\mathrm{cm}^{2} / \mathrm{s} .{ }^{11}$ Watts et $\mathrm{al}^{12}$ measured the thermal diffusivity of some composites, such as Heliomolar $\left(0.0025 \mathrm{~cm}^{2} / \mathrm{s}\right)$, Prisma-Fil $\left(0.0023 \mathrm{~cm}^{2} / \mathrm{s}\right)$, Herculite $\left(0.0028 \mathrm{~cm}^{2} / \mathrm{s}\right)$, Palfique $\left(0.0026 \mathrm{~cm}^{2} / \mathrm{s}\right), \mathrm{P}-10\left(0.0064 \mathrm{~cm}^{2} / \mathrm{s}\right)$, and Belfirm $\left(0.0056 \mathrm{~cm}^{2} / \mathrm{s}\right)$ and observed that they were similar to the thermal diffusivity of dentin.

Organic dyes have been used in dental research since 1933 and have proved to be a valuable tool for assessing marginal infiltration at the restoration interface, as demonstrated by the study of Kanca and Suh ${ }^{13,14}$ in the penetration of a dye into the restorations when they were photopolymerized with lower intensities and compared with a control group whose penetration was even lower when the photoactivation was done by the pulsed form, probably due to a prolonged gel phase, allowing the material to flow. This same view is shared by HelvatjogluAntoniades et al. ${ }^{15}$

According to Bauer and Henson, ${ }^{13}$ the factors that contribute to the penetration of these dyes are the interfacial spaces, inadequate physical properties of the restorative material, and improper restorative technique or procedure. 
Numerous other researches were developed using, as a principle, the evaluation of the penetration of several dyes at the interface of the cavity filled with various materials, and by employing different restorative techniques.

Fluorescence is characterized by the emission of a photon at a wavelength greater than the photon absorbed by matter, and certain dyes, such as rhodamine $6 \mathrm{G}$ have this property.

Light is a form of electromagnetic radiation and its propagation is considered as a wave phenomenon. It is characterized by a wavelength $\lambda$ and a frequency $f$, which are correlated in the equation:

$$
\mathrm{C}=\lambda \times f
$$

A photon, on reaching a fluoresent element, causes a molecular excitation causing this molecule to be transported from a less energetic level (ground state) to a more energetic level (excited state). This excited state is unstable, and the molecule tends to return to its ground state (more stable) through the emission of a photon. However, in the interval between the absorption and emission of the photon, the molecule loses energy by vibrational decays, which result from the interaction between molecules or vibrational changes, and, therefore, do not emit photons. Therefore, when the photon emission finally occurs, there is less energy available, and since the wavelength is inversely proportional to the photon energy, it will have a longer wavelength because the speed of light is constant $(C=300,000 \mathrm{~km} / \mathrm{s})$. Moreover, this speed is dependent on wavelength and frequency $(\mathrm{C}=\lambda \times f)$. With the loss of energy due to molecular excitation, consequently, a decrease in frequency and an increase in wavelength will occur, causing a change in the light emitted. ${ }^{16,17}$

Techniques that use fluorescence as a means of evaluation have been used in several areas of research.

The purpose of this work is to quantify the penetration of the $6 \mathrm{G}$ rhodamine fluorescent dye into a photopolymerized composite resin at several curing temperatures (starting at $0^{\circ} \mathrm{C}$ and going through $25,50,75^{\circ} \mathrm{C}$ to finish with $100^{\circ} \mathrm{C}$ ) by two LEDs (Bright LEC and LEC 1000, MM Optics) and a halogen device (Gnatus Optilight Digital, Gnatus), with activation times of 40 and $60 \mathrm{~s}$. This work attempts to test the hypothesis that these factors influence the penetration of this dye into the composite resin.

\section{MATERIALS AND METHODS}

In this experiment, a composite resin was photopolymerized with different times (40 and $60 \mathrm{~s}$ ) in five different curing temperatures $\left(0,25,50,75\right.$, and $\left.100^{\circ} \mathrm{C}\right)$ by three different light-curing devices, a halogen device called Optilight Digital (Gnatus, Ribeirão Preto, Brazil), a prototype LED device called LEC 1000 developed by MM Optics and referred to as LED 1, and an LED device also developed by MM Optics (São Carlos, Brazil) called Bright LEC and referred to as LED 2. The potencies of these equipment were verified by a specific power meter (Power Meter/FieldMaster, Coherent, Palo Alto, California, USA). The emission spectrum was measured using a USB 2000 spectrophotometer (Ocean Optics, Inc., Dunedin, Florida).

The polymerization was evaluated with the fluorescence spectroscopy to measure the penetration area of a fluorescent dye into a polymerized composite resin. For each of the 30 different situations, 5 repetitions were performed, totaling 150 specimens. The selected material for this study was the Filtek Z250 (3M ESPE/St. Paul, Minnesota). The shade selected was A3.

An aluminum matrix was developed allowing a test specimen of $4 \mathrm{~mm}$ diameter by $4 \mathrm{~mm}$ thickness to be created at the various temperatures proposed (Fig. 1). A thermometer (Minipa, São Paulo) (Fig. 1) was positioned in an orifice to allow the matrix temperature to be verified with a resolution of $0.1^{\circ} \mathrm{C}$.

To reduce the temperature of the matrix to $0^{\circ} \mathrm{C}$, a cooling system was developed using a freezer motor $(7.58 \mathrm{Kw}$, $60 \mathrm{~Hz}, 220 \mathrm{~V}$, Embraco, Joinville, SC). A serpentine was prepared according to the dimensions of the matrix, allowing the insertion and photopolymerization of the composite resin (Fig. 2). The temperature of $25^{\circ} \mathrm{C}$ was considered the ambient temperature controlled by the air conditioner.

For the heating of the matrix at temperatures of 50, 75 , and $100^{\circ} \mathrm{C}$, the matrix was longitudinally crossed with two resistors, which were connected to a voltage regulator (ATV Type 215-M number 2511, STP, São Paulo). By adjusting the voltage, the temperature of the matrix could be raised and controlled based on the measurements of the thermometer inserted into the surface.

The composite resin was inserted with a spatula (PFI 8AL, Cosmedent, Chicago, IL) and then covered with a polyester strip and a glass slide, and a time of $1 \mathrm{~min}$ was

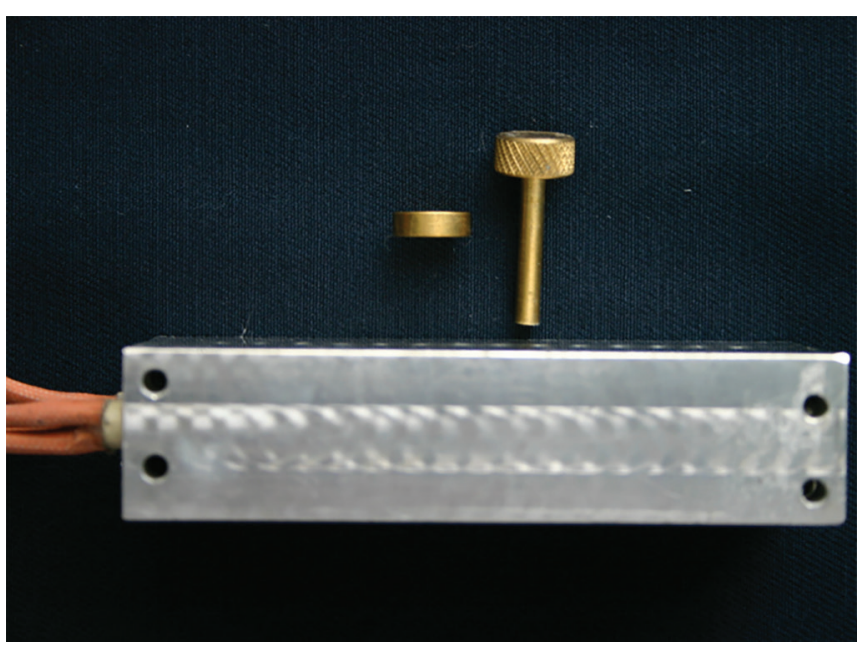

Fig. 1: Aluminum matrix with a pin and a 4-mm spacer. A resistance runs through the entire aluminum matrix 


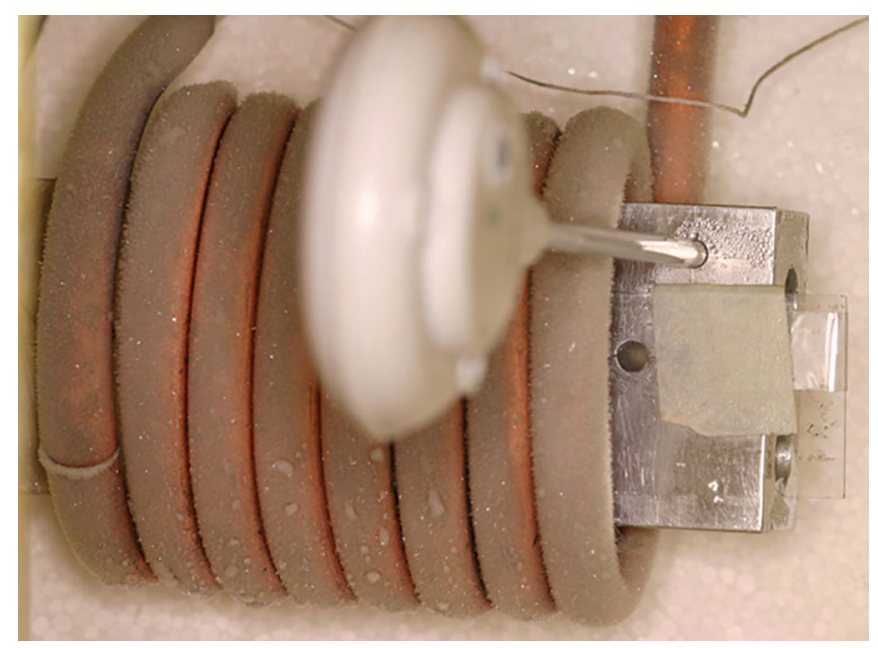

Fig. 2: Matrix with a thermometer positioned inside the serpentine

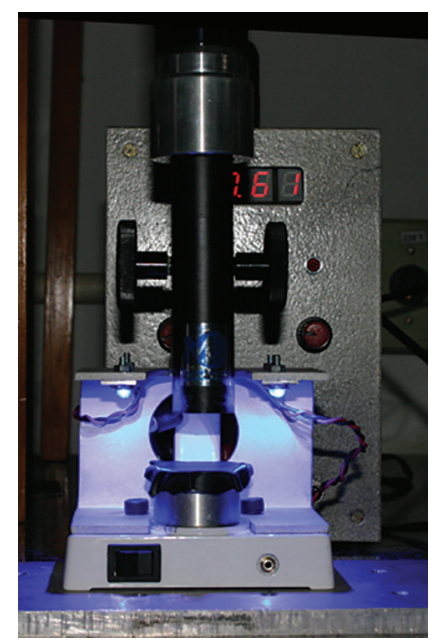

Fig. 4: Monocular optical loupe positioned above the composite with four blue LED illuminators

awaited to let a temperature equalization occur between the matrix and the composite resin.

Immediately after polymerization of the specimen, the sample was inserted into properly labeled Eppendorf tubes containing the rhodamine dye $6 \mathrm{G}$ in $0.1 \%$ ethylene glycol and was left for $24 \mathrm{~h}$. After this period, the specimen was cleansed with distilled water and then dried.

After the specimens were cut, so that they remained with a $1.5 \mathrm{~mm}$ slice and a flat surface (Fig. 3), they were taken to a developed apparatus consisting of a magnifying glass (monocular optical loupe, MM-L1 30×, M\&M Optics, São Carlos) and an illuminator with 4 blue LEDs (Fig. 4) with adjustable power coupled to a digital video camera (charged-coupled device) (GC-405 NG, LG-Honeywell). This camera was connected to a computer through the Matrox PC-VCR program (Québec, Canada), and after the analyses were performed, the images were stored on hard disk.

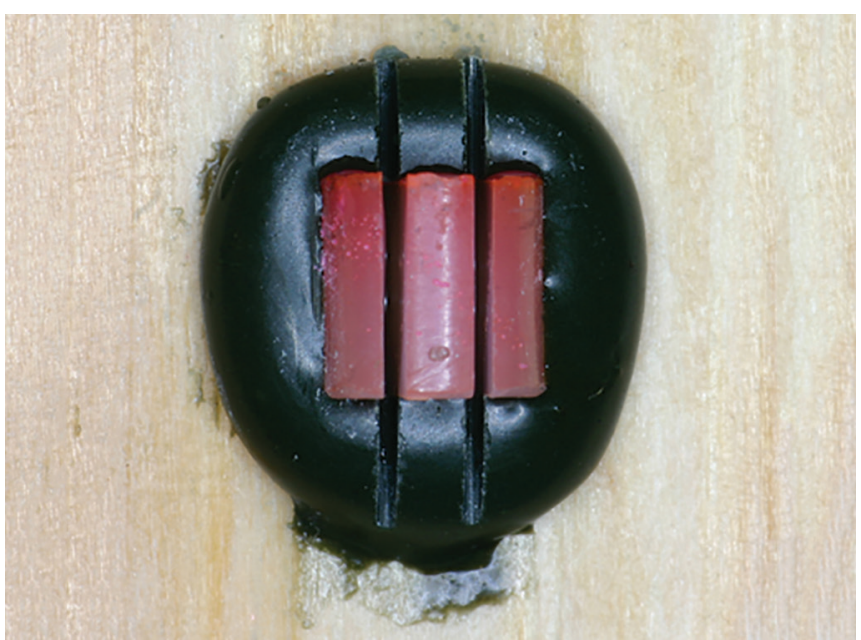

Fig. 3: A $1.5 \mathrm{~mm}$ slice of the composite polymerized and stained with the dye

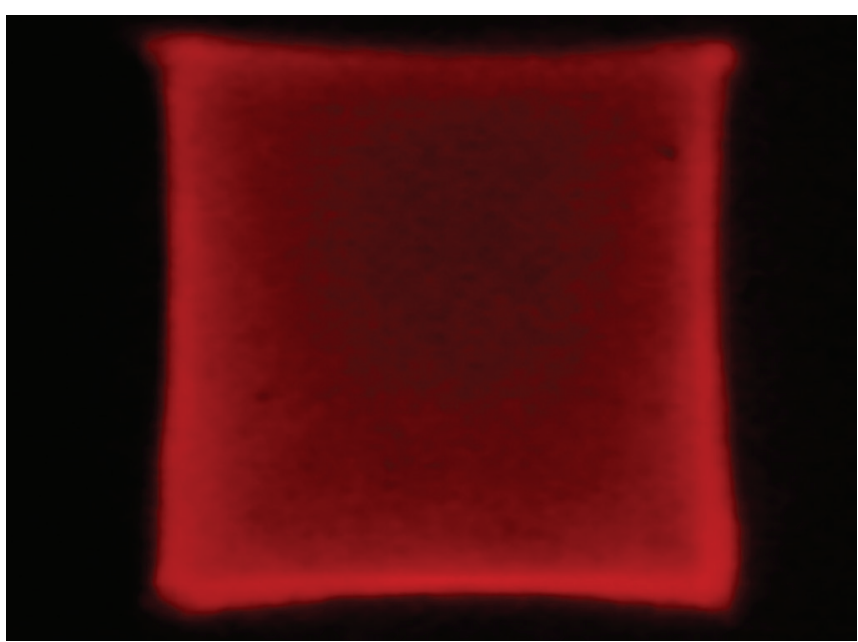

Fig. 5: Mean image of the $\mathrm{H} 40525$ sample (sample number 5 photoactivated with the halogen lamp for $40 \mathrm{~s}$ at $25^{\circ} \mathrm{C}$ ).

The image of each surface was taken 16 times, allowing the average image to be formed (Fig. 5) using an arithmetic mean. A program named Matlab converted the image to grayscale, and each pixel became a value between 0 and 1 . The value 0 means regions without light and is represented by black. On the contrary, the 1 represents regions with the maximum intensity emitted and is represented by white. Values between 0 and 1 are considered changes of gray.

To obtain the results, the percentage of the fluorescent area was calculated according to the following example:

\begin{tabular}{cc} 
Total area (\%) & Total pixel \\
\hline 1 & 1000 \\
$X$ & 500 \\
\hline Fluorescent area (\%) & Fluorescent pixel
\end{tabular}

Therefore, the fluorescent area is:

$$
X=50 \%
$$


That is, $50 \%$ of the sample area was penetrated by rhodamine $6 \mathrm{G}$.

Data were submitted to analysis of variance, Tukey's multiple comparisons test, both tests at $5 \%$ significance level, and Bonferroni correction at $2.5 \%$ significance level.

\section{RESULTS}

Table 1 shows the means and standard deviations of 30 experimental groups. Table 2 describes an analysis of variance summary to assess the effects of photopolymerization apparatus, curing temperatures, photoactivation time, and their interactions on dye penetration percentage. Table 2 also shows p-values to evaluate the homogeneity of variance and residues normality $(p \geq 0.05)$.

Graphs 1 and 2 display the percentage means of fluorescent dye penetration according to the photopolymerization device, curing temperature, and photoactivation, at $95 \%$ confidence intervals.

Individual values, means, and standard deviations showed that dye penetration percentages at 0 and $25^{\circ} \mathrm{C}$ curing temperatures were significantly higher than at 50 ,

Table 1: Means and SD of fluorescent dye penetration percentage according to the photopolymerization devices, curing temperature, and photoactivation time

\begin{tabular}{llllllll}
\hline \multirow{2}{*}{ Devices } & Time & \multirow{2}{*}{$\begin{array}{l}\text { Statistical } \\
\text { analysis }\end{array}$} & \multicolumn{5}{c}{ Curing temperature $\left({ }^{\circ} \mathrm{C}\right)$} \\
\cline { 2 - 7 } & $(\mathrm{s})$ & & 0 & 25 & 50 & 75 & 100 \\
\hline Halogen & 40 & Mean \pm SD & $90.62 \pm 5.03$ & $87.43 \pm 4.98$ & $15.63 \pm 8.78$ & $17.04 \pm 5.86$ & $16.50 \pm 4.71$ \\
& 60 & Mean \pm SD & $87.77 \pm 5.50$ & $69.26 \pm 8.19$ & $11.39 \pm 4.32$ & $14.89 \pm 9.56$ & $12.04 \pm 5.57$ \\
LED 1 & 40 & Mean \pm SD & $88.01 \pm 5.07$ & $86.58 \pm 4.30$ & $35.84 \pm 9.47$ & $13.84 \pm 6.39$ & $29.17 \pm 9.90$ \\
& 60 & Mean \pm SD & $90.99 \pm 3.38$ & $81.54 \pm 4.97$ & $18.35 \pm 10.21$ & $22.96 \pm 10.29$ & $16.60 \pm 10.08$ \\
LED 2 & 40 & Mean \pm SD & $88.64 \pm 4.34$ & $88.19 \pm 4.58$ & $33.39 \pm 8.97$ & $26.18 \pm 8.57$ & $39.47 \pm 8.90$ \\
& 60 & Mean \pm SD & $89.79 \pm 3.62$ & $83.36 \pm 5.91$ & $8.87 \pm 3.55$ & $19.26 \pm 5.50$ & $31.13 \pm 7.32$ \\
\hline
\end{tabular}

SD: Standard deviations

Table 2: Summary of variance analysis of effects and their interactions on dye penetration percentage

\begin{tabular}{llllr}
\hline Variable & Degrees of freedom & Quadratic mean & $f$-value & $p$-value \\
\hline Devices & 2 & 975.05 & 20.03 & $<0.001^{*}$ \\
Time & 1 & 1611.18 & 33.10 & $<0.001^{*}$ \\
Temperature & 4 & 38014.53 & 781.07 & $<0.001^{*}$ \\
Devices $\times$ Time & 2 & 52.71 & 1.08 & 0.342 \\
Devices $\times$ Temperature & 8 & 218.68 & 4.49 & $<0.001^{*}$ \\
Time $\times$ Temperature & 4 & 341.01 & 7.01 & $<0.001^{*}$ \\
Devices $\times$ Time × Temperature & 8 & 147.91 & 3.04 & $0.004^{*}$ \\
Residue & 120 & 48.67 & & \\
\hline
\end{tabular}

Homogeneity of variances: $p=0.050$ (Levene); Residues normality: $p=0.139$ (Shapiro-Wilk); *Significance: $p<0.025$ according to Bonferroni correction

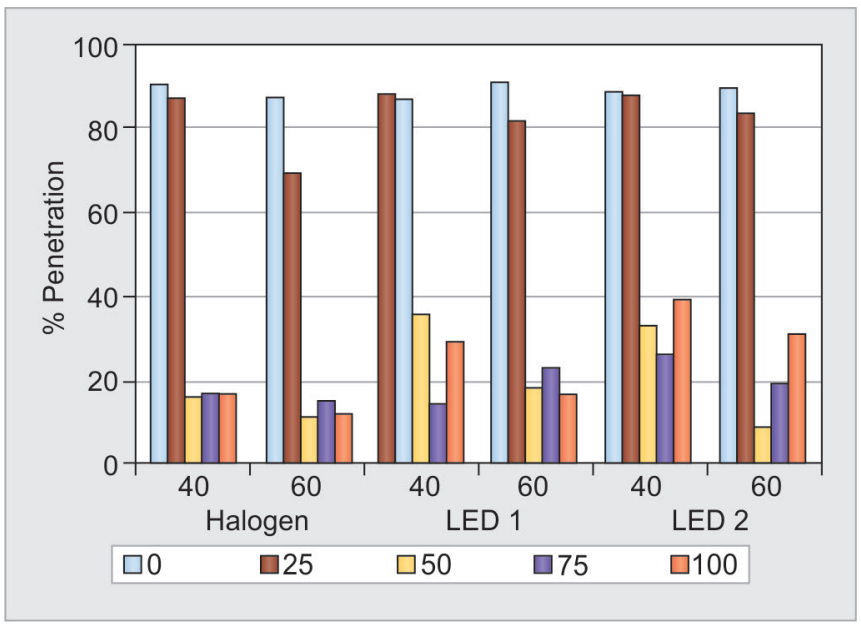

Graph 1: Fluorescent dye penetration percentage means according to different photopolymerization devices and photoactivation times (40 and 60 s). Vertical bars indicate a 95\% confidence interval for population mean

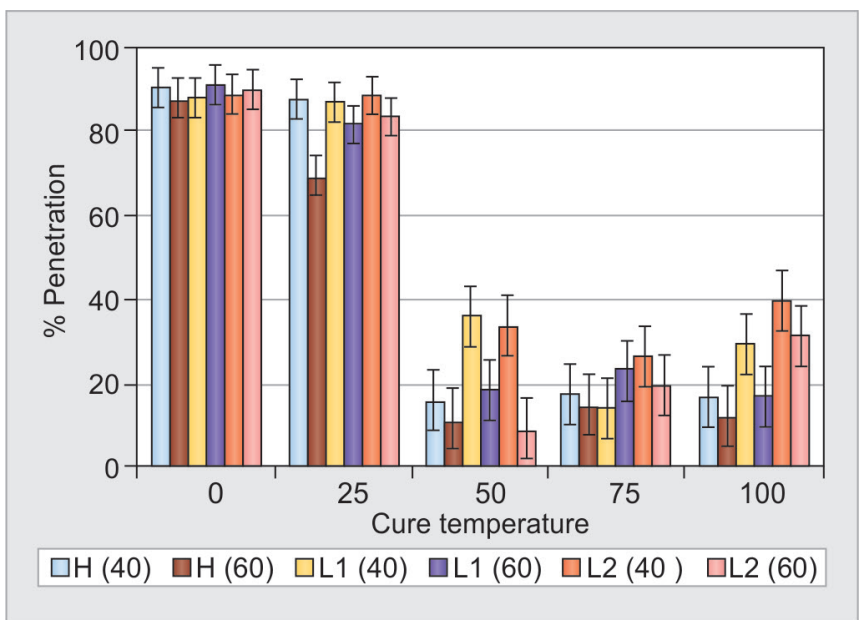

Graph 2: Fluorescent dye penetration percentage means according to curing temperature $\left(0,25,50,75\right.$, and $\left.100^{\circ} \mathrm{C}\right)$. Vertical bars indicate a $95 \%$ confidence interval for population mean 
Table 3: Summary of variance analysis of effects and their interactions on dye penetration percentage at 0 and $25^{\circ} \mathrm{C}$ curing temperatures

\begin{tabular}{llllc}
\hline Variable & Degrees of freedom & Quadratic mean & f-value & $p$-value \\
\hline Devices & 2 & 78.22 & 2.97 & 0.061 \\
Time & 1 & 298.53 & 11.35 & $0.001^{*}$ \\
Temperature & 1 & 648.55 & 24.66 & $<0.001^{*}$ \\
Devices $\times$ Time & 2 & 138.24 & 5.26 & $0.009^{*}$ \\
Devices $\times$ Temperature & 2 & 73.43 & 2.79 & 0.071 \\
Time $\times$ Temperature & 1 & 358.29 & 13.62 & $0.001^{*}$ \\
Devices $\times$ Time $\times$ Temperature & 2 & 30.16 & 1.15 & 0.326 \\
Residue & 48 & 26.30 & & \\
\hline
\end{tabular}

Homogeneity of variances: $p=0.504$ (Levene); residues normality: $p=0.057$ (Shapiro-Wilk); *Significance: $p<0.025$ according to Bonferroni correction

Table 4: Summary of variance analysis of effects and their interactions on dye penetration percentage at 50,75 , and $100^{\circ} \mathrm{C}$ curing temperatures

\begin{tabular}{llllc}
\hline Variable & Degrees of freedom & Quadratic mean & $f$-value & $p$-value \\
\hline Devices & 2 & 1097.77 & 17.26 & $<0.001^{*}$ \\
Time & 1 & 1422.22 & 22.37 & $<0.001^{*}$ \\
Temperature & 2 & 207.15 & 3.26 & 0.044 \\
Devices $\times$ Time & 2 & 179.80 & 2.83 & 0.066 \\
Devices $\times$ Temperature & 4 & 300.17 & 4.72 & $0.002^{*}$ \\
Time $\times$ Temperature & 2 & 448.08 & 7.05 & $0.002^{*}$ \\
Devices $\times$ Time $\times$ Temperature & 4 & 148.07 & 2.33 & 0.064 \\
Residue & 72 & 63.58 & & \\
\hline
\end{tabular}

Homogeneity of variances: $p=0.296$ (Levene); residues normality: $p=0.052$ (Shapiro-Wilk); *Significance: $p<0.025$ according to Bonferroni correction

75 , and $100^{\circ} \mathrm{C}$. Two tests of variance were performed: One for the first two curing temperatures (Table 3), and the other one for the last three curing temperatures, as shown in Table 4 . These analyses were complemented by Tukey's multiple comparisons test. Assumptions of homogeneity of variance and residues normality were considered valid.

Regarding curing temperatures $\left(0\right.$ and $\left.25^{\circ} \mathrm{C}\right)$, according to the analysis of variance summary, photoactivation time and temperature parameters presented significant values $(p<0.05)$ and showed that, at $25^{\circ} \mathrm{C}$, the percentage of dye penetration was lower if a light curing was performed for $60 \mathrm{~s}$. Different devices did not present significant differences, but p-value was slightly higher than the 0.025 significant level. On the contrary, parameters interactions showed the relation between photopolymerization device and photoactivation time and between temperature and photoactivation time. Regardless of the curing temperature, halogen lamp and $60 \mathrm{~s}$ of photoactivation showed lower dye penetration percentage (10\% lower). Regardless of the device, $60 \mathrm{~s}$ of photoactivation and $25^{\circ} \mathrm{C}$ showed that dye penetration percentage was $10 \%$ lower than the other groups.

Regarding curing temperature results $(50,75$, and $100^{\circ} \mathrm{C}$ ) showed in Table 4, device and time were significantly high, while temperature was not significant, $\mathrm{p}$-value was close to 0.05 . After $60 \mathrm{~s}$ of light curing, a lower penetration occurred and tended to increase at $100^{\circ} \mathrm{C}$.
Regardless of the photoactivation time, dye penetration percentage means were higher at $100^{\circ} \mathrm{C}$ curing temperature, except in LED 1 at $50^{\circ} \mathrm{C}$, which was significantly higher than halogen percentage means.

At 50 and $100^{\circ} \mathrm{C}$ and $40 \mathrm{~s}$ of photopolymerization, parameters presented the highest penetration percentages means. However, the lowest mean was obtained at $60 \mathrm{~s}$ of photoactivation with a curing temperature of $50^{\circ} \mathrm{C}$, and despite been lower than the other ones, it was not significant.

\section{DISCUSSION}

A high-temperature environment generates polymers with certain characteristics: A higher compressive strength; a diminished permeability to chemical agents due to an altered diffusion pattern, and a reduction of the diffusion rate of penetrants; a high Knoop hardness, ${ }^{5}$ a better degree of conversion as seen in evaluations performed in a differential thermal analyzer; ${ }^{18}$ and in FTIR spectroscopy: ${ }^{19-23}$ a high resistance to folding, ${ }^{20}$ a high initial reaction rate and consequently an initial free excess volume that improves the final conversion degree and decreases contraction stress generated; $; 1,24,25$ an increase in the maximum conversion rate ${ }^{22}$ as well as a higher conversion at the maximum rate and a delay in self-deceleration during the cure process ${ }^{26}{ }^{26}$ increase in 
the glass transition temperature $(\mathrm{Tg})$ of the polymerized resins; ${ }^{25}$ and greater structural heterogeneity. ${ }^{27}$

Light-initiated polymerizations have a high initial reaction rate. ${ }^{24}$ However, with the continuity of the reaction, the mobility of the reactive species becomes even smaller and the propagation of the reaction becomes diffuse-limited, i.e., even if there are free radicals and monomers, they cannot react because they cannot move within the mass reactant. This phenomenon is called selfdeceleration and is responsible for the considerable drop in reaction rate. Thus, the final conversion of polymerization will be controlled by the diffusion limitations of the reagent medium and not by the amount of free monomers in the system.

By raising the curing temperature during the photopolymerization, it is possible to delay the process of selfdeceleration because of the decrease in the viscosity of the reagent system and an increase in the monomeric motility. The reaction continues for longer periods because there is an increase in the motility before the propagation process becomes diffuse-limited, allowing the final conversion to be increased..$^{25-27}$

It is clear that these changes depend not only on the amount of heat applied in the samples, but also on the configuration of the resinous systems used, the incorporation or not of loads, the form of analysis used in the study, the photopolymerizer, and the time of photoactivation.

In glassy polymers, the diffusion rate of penetrants is related with the free volume of the reaction, and it is also dependent on the degree of polymerization. When an increase in the degree of polymerization occurs, a decrease in free volume will occur too, and therefore, the delay in the diffusion process. ${ }^{28}$ Thus, the penetration of the rhodamine dye $6 \mathrm{G}$ in the polymer mass depends on the free volume. Related to macromolecular materials and according to Kumins and Kwei, ${ }^{28}$ the effectiveness of the polymerization reagents as well as the physical and chemical characteristics of the polymer produced are related to the mobility of small molecules.

A mathematical model of diffusion in polymers was described by Vrentas and Duda, ${ }^{29,30}$ and it states that the free volume within a system is responsible for controlling the molecular transport. Therefore, the greater the penetration of the dye is, the larger is the free volume. In addition to the degree of conversion, the free volume is also dependent on the form of polymerization. Soh and Yap ${ }^{31}$ verified that photopolymerizations performed through the late pulse could result in more linear structures with lower cross-link density, resulting in a higher free volume of the material, since these polymers had a lower glass transition temperature, due to their cross-link density, intermolecular interactions (Van der Waals and
London forces), and the degree of conversion. TruffierBoutry et $\mathrm{al}^{32}$ believed that the postphotopolymerization contraction is due to the decrease in free sample volume due to the relaxation achieved by the molecules in the search for a more stable condition and not a postcure conversion as usually reported.

A high cross-link density characterizes the formation of microgels of composite resin. As described by Rey et $\mathrm{al}_{,}{ }^{33}$ they can be divided schematically into two parts: One would be its core characterized as an inaccessible part, with a dense network of cross-links and consequently a high $\mathrm{Tg}$; and the other part would be its outer portion where a small amount of cross-links exist, determining a low $\mathrm{Tg}$. Reactive species located in this region may diffuse and react with other microgels. Based on this study, it is worth to say that there is no penetration of dye inside the microgel, but around it, which proves the theory of diffusion in the region of the free volume of the polymer.

It is expected that there would be a higher dye penetration at $0^{\circ} \mathrm{C}$ than at $25^{\circ} \mathrm{C}$, because higher the volume, the greater the conversion, and the lower the free volume, ${ }^{22,23}$ which, in turn, would be greater at $50^{\circ} \mathrm{C}$ and even more than at 75 and $100^{\circ} \mathrm{C}$. However, as can be seen, two sets of results could be distinguished.

A first group comprises the photopolymerized samples at curing temperatures of 0 and $25^{\circ} \mathrm{C}$, which showed a much higher penetration of dye in relation to the other Tcure evaluated. Within this group, the dye penetration was lower at $25^{\circ} \mathrm{C}$, and when a longer activation time (60 s) was used. There was no statistically significant difference between the devices, although the halogen apparatus tended to have a lower penetration average than the other two devices, perhaps justifying Asmussen and Peutzfeldt's ${ }^{34}$ assertion that the importance of temperature is higher in regions of lower polymerization or in the case as in a situation where we would probably have a lower conversion as the Tcure of $0^{\circ} \mathrm{C}$. The halogen apparatus generated more heat due to the irradiance, there was less penetration of the dye with $60 \mathrm{~s}$ of photoactivation. The other group would comprise curing temperatures of 50,75 , and $100^{\circ} \mathrm{C}$, where we can observe a severe decrease in the penetration of the dye in relation to $25^{\circ} \mathrm{C}$. At some curing temperature value between 25 and $50^{\circ} \mathrm{C}$, there may be an onset of energy supply, which is sufficient to agitate the molecules in such a way that they react more and more, increasing the degree of conversion and cross-links, even with the increased viscosity resulting from the conversion. Considering the three higher values, the Tcure of $50^{\circ} \mathrm{C}$ was the one that better prevented the penetration of the dye on average, although in some situations, Tcure of $75^{\circ} \mathrm{C}$ showed a better behavior. In the Tcure of $50^{\circ} \mathrm{C}$, the penetration average was lower with $60 \mathrm{~s}$ independent of the photopolymerizer. What is speculated is that above 
the curing, temperature of $50^{\circ} \mathrm{C}$, evaporation of components may occur. Another hypothesis that could be cited is the change of the light transmission toward the deeper parts of the sample due to an extremely rapid conversion in the upper portions due to a great acceleration in the reaction rate.

The generation of heat during the conversion of monomer into polymers will cause modifications in the cure kinetics. This increase in temperature, however, depends on the sum of two factors: Exotherm of the reaction and irradiance of the photopolymerizer. The amount of heat generated also depends on the polymer cure rate, which is dependent on several factors, such as transmission of light within the composite, light source emission spectrum, sample thickness, absorption spectrum, light intensity, polymerization form (ramp, soft-start, and pulsed), and type and amount of charge. 8,26

In light of polymerization effectiveness, it may be advantageous for the heat-generating apparatus to be in the depth of polymerization or in the conversion degree in deeper regions because, and due to the rapid conversion of the upper regions, there may not be sufficient time for the dissipation heat to influence healing. Already in a deeper layer, there is the possibility of heating before the end of the reaction. This may benefit the halogen devices, especially those like the one used in this work, which has a relatively moderate intensity and which generates more heat than the other two LEDs. As reported by Uhl et al, ${ }^{35}$ using infrared camera, the heat dissipation is fast into the composite, and with about $10 \mathrm{~s}$, the peak is already reached. However, Hansen and Asmussen ${ }^{36}$ consider that a small increase in the depth of polymerization is followed by a disproportionate increase in temperature. It is important to consider that, in the same depth, there are differences in temperature as demonstrated by Al-Qudah et $\mathrm{al}^{37}$ who verified, also using infra-red camera, higher temperature increases of the composite resin in its central portion during cure.

In a comparison, the heat from irradiation of the apparatus and the exotherm of the composite resin were in many cases similar when using the halogen lamps. However, with the LED-based system, the greatest increase occurred by the exotherm and not by the irradiation, demonstrating that, due to its emission spectrum, the cure rate can be high. ${ }^{38}$

Hofmann et $\mathrm{a}^{38}$ demonstrated through the analysis of the polymerization contraction that, during curing of composites by halogen lamps, two phenomena occur simultaneously: One would be the contraction resulting from the reaction and the other the expansion caused by the generation of heat. After photoactivation, a slight increase in the contraction rate was observed, demonstrating that the reaction is occurring in an expanded state.
With the LEDs, this phenomenon was not observed due to the lower heat generation of the devices used in the study. This phenomenon was also reported by Mondelli et al. ${ }^{39}$ However, in a recent study, ${ }^{40}$ LEDs with an intensity of $800 \mathrm{~mW} / \mathrm{cm}^{2}$ (FreeLight 2, 3M ESPE) and $1100 \mathrm{~mW} / \mathrm{cm}^{2}$ (Bluephase, Ivoclar Vivadent) produced an increase in contraction rate similar to a halogen lamp.

One characteristic of the photopolymerizable restorative system is its rapid gelification with only $5 \%$ of the gel point being reached. ${ }^{41}$ According to Lange et al, ${ }^{42}$ the gel point is a well-characterized, distinct, and nonreversible event, defined as the appearance of an insoluble fraction of polymer involving an entire continuous structural network extending throughout the macroscopic dimension of the specimen.

After this event, the elastic limit of the composite resin reaches a certain level where the increase in stiffness does not allow enough plastic deformation to compensate for the volume reduction due to the polymerization contraction. ${ }^{43}$ According to Dickens et al, ${ }^{44}$ one of the main factors to obtain a good reactivity would be a low initial viscosity of the resin.

The kinetics of the reaction is significantly influenced by the supply of thermal energy before the initiation of photopolymerization. The modification of the polymerization process will modify the physicochemical properties of the polymer and its arrangement. The curing temperature can be included as an influencer of the curing process along with other factors already cited. Subtle elevation in the temperature of the nonpolymerized composite was able to modify the properties of the materials when polymerized. ${ }^{20,23,45}$

\section{CONCLUSION}

The modification of curing temperature of dental composites, consequently, will alter the kinetics of the reaction, the structural morphology, and the physicochemical characteristics of the composite resin. However, for these phenomena to occur, the rate of reaction must be slow enough so that no vitrification of the polymer occurs and probably entrapment of monomers and free radicals in inaccessible locations will not occur either.

It is evident, based on our results and data available, that small increments of heat increased the degree of conversion, and that the heat generated by the light used for photoactivation can induce a heating in the reagent medium monomer by reducing its viscosity and increasing the mobility and agitation of its components.

With the vast intensity, the LED 2 demonstrated a more homogeneous polymerization. This can be attributed to its rate of polymerization that provides polymers not too long and consequently with a bigger free volume, 
and this rate is not fast enough to cause the reagents to entrap themselves or block the passage of light into the rapidly converted surface layers. This justifies why this device demonstrated the lowest mean penetrance compared with all situations that were exploited.

New experiments must be carried out to evaluate the effects of the heating on the monomer before its photoactivation, and it does not matter if this heat comes from the environment, the light source, or the exothermic reaction. A larger investigation must be performed to analyze these phenomena related to physical properties and polymerization kinetics.

\section{Clinical Significance}

The dentist must be aware of the effects that exist between activation devices regarding the light output and their heat transmission to the composite and the tooth itself. This heat transmission might create a polymer with better characteristics.

\section{ACKNOWLEDGMENT}

Authors would like to thank CePOF-IFSC/Usp São Carlos.

\section{REFERENCES}

1. Mills RW. Blue light emitting diodes-another method of light curing? Br Dent J 1995 Mar;178(5):169.

2. FujibayashiI K, Ishimaru K, Takahashi N, Kohno A. Newly developed curing unit using blue light emitting diodes. Dent Jpn 1998 Mar;34:49-53.

3. Uhl A, Mills RW, Jandt KD. Optical power outputs, spectra and dental composite depths of cure, obtained with blue light emitting diode (LED) and halogen light curing units (LCUs). Br Dent J 2002 Oct;193:459-463.

4. Uhl A, Sigusch BW, Jandt KD. Second generation LEDs for the polymerization of oral biomaterials. Dent Mater 2004 Jan;20(1):80-87.

5. Wu, WI. Wear mechanisms of dental composite resins. In: Leinfelder KF, Taylor DF, editors. Posterior composites-proceedings of the international symposium on posterior composite resins. North Carolina: Chapel Hill; 1984. pp. 127-146.

6. De Gee AJ, ten Harkel-Hagenaar E, Davidson CL. Color dye for identification of incompletely cured composite resins. J Prosthet Dent 1984 Nov;52(5):626-631.

7. Andrade, LE. Estudo da difusão de um corante orgânico em resinas compostas polimerizadas com duas fontes de luz: Avaliação através de espectroscopia de fluorescência. 2004. 116 f. Universidade Estadual Paulista: Tese (Doutorado em Dentística Restauradora)-Faculdade de Odontologia; 2004.

8. Lloyd $\mathrm{CH}$, Joshi A, McGlynn E. Temperature rises produced by light sources and composites during curing. Dent Mater 1986 Aug;2(4):170-174.

9. Watts DC. Reaction kinetics and mechanics in photopolymerised networks. Dent Mater 2005 Jan;21(1):27-35.

10. Anusavice, KJ. Phillips materiais dentários. 10th ed. Rio de Janeiro: Guanabara Koogan; 1998.
11. Brown WS, Dewey WA, Jacobs HR. Thermal properties of teeth. J Dent Res 1970 Jul-Aug;49(4):752-755.

12. Watts DC, McAndrew R, Lloyd CH. Thermal diffusivity of composite restorative materials. J Dent Res 1987 Oct;66(10):1576-1578.

13. Bauer JG, Henson JL. Microleakage: a measure of the performance of direct filling materials. Oper Dent 1984 Winter;9(1):2-9.

14. Kanca J 3rd, Suh BI. Pulse activation: Reducing resin-based composite contraction stresses at the enamel cavosurface margins. Am J Dent 1999 Jun;12(3):107-112.

15. Helvatjoglu-Antoniades M, Kalinderis K, Pedulu L, Papadogiannis $Y$. The effect of pulse activation on microleakage of a 'packable' composite resin and two 'ormocers'. J Oral Rehabil 2004 Nov;31(11):1068-1074.

16. Guilbault, GG. Introduction to luminescence. In: Guilbault GG, editor. Pratical fluorescence-theory, methods and techniques. New York: Marcel Dekker; 1973. pp. 1-17.

17. Bassani, F. Luminescence: General concepts and applications to the study of solids. In: Jameson DM, Reinhart GD, editors. Fluorescent biomolecules methodologies and applications. New York: Plenum Press; 1989. pp. 1-7.

18. McCabe JF. Cure performance of light-activated composites by differential thermal analysis (DTA). Dent Mater 1985 Dec;1(6):231-234.

19. Bennett AW, Watts DC. Performance of two blue lightemitting-diode dental light curing units with distance and irradiation-time. Dent Mater 2004 Jan;20(1):72-79.

20. Li J, Nicander I, von Beetzen M, Sundström F. Influence of paste temperature at curing on conversion rate and bending strength of light-cured dental composites. J Oral Rehabil 1996 May;23(5):298-301.

21. Scherzer T, Decker U. The effect temperature on the kinetics of diacrylate photopolymerizations studied by real-time FTIR spectroscopy. Polymer 2000 Oct;41:7681-7690.

22. Trujillo M, Newman SM, Stansbury JW. Use of near-IR to monitor the influence of external heating on dental composite photopolymerization. Dent Mater 2004 Oct;20(8):766-777.

23. Daronch M, Rueggeberg FA, De Goes MF. Monomer conversion of pre-heated composite. J Dent Res 2005 Jul;84(7): 663-667.

24. Lecamps L, Youssef B, Bunel C, Lebaudy P. Photoinitiated polymerization of a dimethacrylate oligomer: 1 . Influence of photoinitiator concentration, temperature and light intensity. Polymer 1997 Dec;38(25):6089-6096.

25. Lovell LG, Lu H, Elliott JE, Stansbury JW, Bowman CN. The effect of cure rate on the mechanical properties of dental resins. Dent Mater 2001 Nov;17(6):504-511.

26. Lovell LG, Newman SM, Bowman CN. The effects of light intensity, temperature, and comonomer composition on the polymerization behavior of dimethacrylate dental resins. J Dent Res 1999 Aug;78(8):1469-1476.

27. Lu H, Lovell LG, Bowman CN. Exploiting the heterogeneity of cross-linked photopolymers to create high- $\mathrm{T}_{\mathrm{g}}$ polymers from polymerizations performed at ambient conditions. Macromolecules 2001 Oct;34(23):8021-8025.

28. Kumins, CA.; Kwei, TK. Free volume and other theories. In: Crank J, Park GS, editors. Diffusion in polymers. New York: Academic Press; 1968. pp. 107-125.

29. Vrentas JS, Duda JL. Diffusion in Polymer-solvent systems. I - Reexamination of the free volume theory. J Polymer Sci 1977 Mar;15(3):403-416. 
30. Vrentas JS, Duda JL. Diffusion in polymer-solvent systems. II - A predictive theory for the development of diffusion coefficients on temperature, concentration and molecular weight. J Polymer Sci 1977 Mar;15(3):417-439.

31. Soh MS, Yap AU. Influence of curing modes on crosslink density in polymer structures. J Dent 2004 May;32(4):321-326.

32. Truffier-Boutry D, Demoustier-Champagne S, Devaux J, Biebuyck JJ, Mestdagh M, Larbanois P. A physico-chemical explanation of the post-polymerization shrinkage in dental resins. Dent Mater 2006 May;22(5):405-412.

33. Rey L, Duchet J, Galy J, Sautereau H, Vouagner D, Carrion L. Structural heterogeneities and mechanical properties of vinyl/ dimethacrilate networks synthesized by thermal free radical polymerization. Polymer 2002 Jul;43:4375-4384.

34. Asmussen E, Peutzfeldt A. Influence of specimen diameter on the relationship between subsurface depth and hardness of a light-cured resin composite. Eur J Oral Sci 2003 Dec;111(6):543-546.

35. Uhl A, Mills RW, Jandt KD. Polymerization and light-induced heat of dental composites cured with LED and halogen technology. Biomaterials 2003 May;24(10):1809-1820.

36. Hansen EK, Asmussen E. Correlation between depth of cure and temperature rise of a light-activated resin. Scand J Dent Res 1993 Jun;101(3):176-179.

37. Al-Qudah AA, Mitchell CA, Biagioni PA, Hussey DL. Thermographic investigation of contemporary resin-containing dental materials. J Dent 2005 Aug;33(7):593-602.
38. Hofmann N, Hugo B, Klaiber B. Effect of irradiation type (LED or QTH) on photo-activated composite shrinkage strain kinetics, temperature rise, and hardness. Eur J Oral Sci 2002 Dec;110(6):471-479.

39. Mondelli RF, Cavina, DA, Espinosa JCC, Franco EB, Mondelli J. Avaliação das forças geradas durante a contração de polimerização de resinas compostas híbridas. JBD: Rev Ibero-Am Odontol Estet Dent Oper 2003 Jul-Sep;27(7):238-245.

40. Ilie N, Felten K, Trixner K, Hickel R, Kunzelmann KH. Shrinkage behavior of a resin-based composite irradiated with modern curing units. Dent Mater 2005 May;21(5):483-489.

41. Stansbury JW, Trujillo-Lemon M, Lu H, Ding X, Lin Y, Ge J. Conversion-dependent shrinkage stress and strain in dental resins and composites. Dent Mater 2005 Jan;21(1): 56-67.

42. Lange J, Altmann N, Halley P, Kelly CT. Understanding vitrification during cure of epoxy resins using dynamic scanning calorimetry and rheological techniques. Polymer 2000Jul;41(15):5949-5955.

43. Braga RR, Ferracane JL. Alternatives in polymerization contraction stress management. J Appl Oral Sci 2004;12(Spe):1-11.

44. Dickens SH, Stansbury, JW, Choi KM, Floyd JE. Photopolymerization kinetics of methacrilate dental resins. Macromolecules 2003 Aug;36(16):6043-6053.

45. Bandyopadhyay S. A study of the volumetric setting shrinkage of some dental materials. J Biomed Mater Res 1982 Mar;16(2):135-144. 